\title{
Desarrollo de Competencias Docentes en Educación Infantil. Una Experiencia Interdisciplinar en la Formación Inicial de Profesores
}

\author{
Carmen Santos-González y Carmen Sarceda-Gorgoso* \\ Facultad de Formación del Profesorado, Dpto. de Pedagogía y Didáctica, Universidad de Santiago de \\ Compostela. Avda. Ramón Ferreiro, s/n, 27002, Lugo-España \\ (e-mail: mcarmen.santos@usc.es; carmen.sarceda@usc.es)
}

Recibido Abr. 4, 2017; Aceptado Jun. 21, 2017; Versión final Ago. 21, 2017, Publicado Dic. 2017

\begin{abstract}
Resumen
Este trabajo pretende dos objetivos: aproximarse a la percepción global del desarrollo de las competencias en las materias que integran una experiencia de innovación interdisciplinar en la formación inicial de profesores; y realizar un análisis diferencial comparativo entre la situación antes y después de la experiencia. La formación inicial del profesorado es actualmente un tema de especial atención en el seno de la investigación educativa, en tanto que es entendida como un elemento esencial para el desarrollo de una enseñanza de calidad. En la investigación, se opta por un diseño pretest y postest que permita medir el cambio en la autopercepción del alumnado con relación a su perfil competencial antes y después de la experiencia interdisciplinar. Se trata de un estudio cuantitativo basado en la técnica de encuesta, de corte no experimental y en el que no hubo manipulación de las variables independientes. Las competencias vinculadas a las materias integradas en el proyecto se desarrollan considerablemente a juicio del alumnado.
\end{abstract}

\section{Development of Teaching Skills in Early Childhood Education. An Interdisciplinary Experience in the Initial Training of Teachers}

\begin{abstract}
This work has two objectives: approaching the global perception of the development of competencies in matters belonging to interdisciplinary innovation experience in the initial training of teachers; and perform a differential analysis between the situation before and after the experience. The initial training of teachers is nowadays a subject that receives especial attention among educators and is understood as an essential element to reach quality teaching. A pretest and posttest design was chosen for the study, design that allows measuring the change in the self-perception of students in relation to their competence profile before and after the interdisciplinary experience. This is a quantitative study based on the technical of survey, of not experimental type and in which there was no manipulation of the independent variables. The results allowed checking that competences linked to the subjects integrated in the project are developed considerably in view of students. As conclusion we can highlight the interdisciplinary project has contributed to the development of the purported competences.
\end{abstract}

Keywords: competences; childhood education; research; educational innovation; interdisciplinary approach. 


\section{INTRODUCCIÓN}

La formación inicial del profesorado es actualmente un tema de especial atención en el seno de la investigación educativa, en tanto que es entendida como un elemento esencial para el desarrollo de una enseñanza de calidad, reflejándose esta importancia en los numerosos estudios llevados a cabo en las últimas décadas (Barber y Mourshed, 2007, European Comission, 2010; EURYDICE y CEDEFOP, 1995; OCDE, 2014, entre otros). Avalos (2011) realiza una revisión en profundidad de 111 publicaciones científicas en revistas de impacto en el ámbito internacional entre los años 2000 y 2010, concluyendo que el desarrollo profesional de los maestros es estudiado y presentado en la literatura de muchas maneras diferentes en las que aparece un elemento común a todas: el desarrollo profesional docente es un proceso complejo que requiere además una implicación cognitiva y emocional.

Ligada al desarrollo profesional está la actividad reflexiva (Desimone, 2011; Fernández-Fernández et al., 2016) como un elemento necesario para superar el carácter reproductor de la actividad docente y adoptar un rol activo que potencie y permita la creación y la innovación. Se pone de manifiesto cómo las escuelas pueden crear poderosas experiencias de desarrollo profesional (Darling-Hammond y Richardson, 2009) y la influencia del modelo experimentado por el maestro en sus procesos de formación en cambios en las prácticas profesionales (Goodwin y Kosnik, 2013) y en los procesos de enseñanza-aprendizaje que diseña y desarrolla con su alumnado. Como consecuencia, se argumenta la necesidad de un nuevo enfoque en la formación del profesorado que amplíe las oportunidades de aprendizaje (Zeichner, 2010), de manera que la educación en general, y la formación de su profesorado en particular se considera, en el contexto de políticas sociales avanzadas, una estrategia necesaria para mejorar los problemas sociales y el crecimiento nacional (Goodwin y Kosnik, 2013).

En las últimas décadas, casi todos los países han iniciado algún tipo de reforma dirigida a mejorar la calidad de la educación (Girvan et al., 2016), siendo este concepto -la calidad-, un término no exento de divergencias y que suscita discusión, tal y como se constata en la literatura sobre el tema (AlvaradoLagunas et al., 2015; Avalos, 2011; Renta-Davids et al., 2016), debido sobre todo a su naturaleza intangible. Sin embargo, pese a la dificultad de conceptualizar unámimemente el término calidad, dada la complejidad del problema al incluir elementos de valor difícilmente observables y la confluencia de múltiples variables, factores y agentes, es posible constatar una serie de indicadores consensuados sobre los que pivotaría la calidad, siendo uno ellos el profesorado. De esta manera, se pone especial énfasis en la necesidad de repensar la formación del profesorado, de manera que los futuros docentes adquieran protagonismo en su proceso de desarrollo profesional y se conviertan en agentes activos del proceso. Esto les facilitaría y permitiría poder llevar a cabo sus funciones desde parámetros caracterizados por la flexibilidad, adaptación y cambio, en el contexto de un proceso de ajuste a los tiempos, las necesidades, las estructuras y las personas.

Al mismo tiempo, se reivindica una Europa del conocimiento que permita "acabar con las fronteras educativas y desarrollar un marco de enseñanza y aprendizaje que favorezca la movilidad y cooperación en el seno de la Unión" (López et al., 2016). Conforme a este desafío, los distintos países comienzan un proceso de cambio y renovación en sus instituciones de educación superior y que en el caso de España se concreta en cuatro dimensiones básicas: a) discente, b) docente, c) curricular, d) organizativo. Se apuesta por la incorporación de un enfoque educativo en el que el eje central es el aprendizaje por competencias, y que exige centrar los procesos en el estudiante, la incorporación escenarios flexibles y abiertos que potencien la formación y el aprendizaje, o establecer nuevas formas de evaluación y/o heteroevaluación, entre otros aspectos (Ausín et al., 2016).

Pero, tal y como sucedía con la calidad, el concepto de competencias tampoco está exento de polémica debido a su carácter polisémico y a la diferente percepción que tiene cada uno de los implicados: estudiantes, docentes, comités....(Villaroel y Bruna). Ello obliga a realizar enormes esfuerzos en la línea de ofrecer una conceptualización consensuada del término, así como en definir qué competencias profesionales deberían desarrollar los futuros titulados (Sarceda-Gorgoso y Rodicio García, en prensa), siendo la cuestión especialmente compleja cuando el foco de atención se sitúa en la competencia docente. Teniendo en cuenta estas limitaciones, en este trabajo adoptamos una conceptualización amplia de término, entendiendo que las competencias profesionales implican necesariamente dimensiones complementarias referidas a capacidades en el ámbito del saber, saber hacer y saber actuar, y todo ello en el marco de los contextos específicos del ejercicio profesional que son los que dan sentido, al mismo tiempo que condicionan, las competencias profesionales.

Esta importancia otorgada al enfoque por competencias en la educación superior tiene reflejo en la literatura especializada, tanto en lo que respecta a la universidad globalmente considerada, como a las titulaciones de Educación y a la formación inicial de maestros, de manera más específica, que comienzan a aplicar este 
enfoque formativo (Icarte y Labate, 2016). Esto conlleva una profusión de estudios e investigaciones que abordan diferentes temáticas en los que subyace el reconocimiento de que el aprendizaje y el desarrollo de los maestros es un proceso complejo que reúne una gran cantidad de elementos diversos y se caracteriza por un acervo de factores igualmente importantes (Avalos, 2011) y que sugieren que existe un conjunto básico de características comunes al desarrollo profesional eficaz y que proporcional un punto de partida desde el cual los investigadores pueden evaluar su efectividad (Girvan et al., 2016).

Así, es posible encontrar estudios que profundizan en diferentes aspectos. Con respecto a la figura del mentor y en las oportunidades de aprendizaje individualizado que ofrecen a los estudiantes, a través de una metodología de grupos focales, Davis y Jacobsen (2014) ofrecen sugerencias prácticas para otras instituciones que tratan de poner en práctica iniciativas de cambio innovadoras en general, y para ampliar las oportunidades para la investigación de grado y actividades creativas en particular. Otro aspecto fundamental es la vinculación entre conocimiento teórico y experiencia práctica, abordado por De la Cerda (2014) a través del aprendizaje-servicio con la finalidad de contribuir a la mejora de la formación inicial de los estudiantes universitarios. Por otra parte, y tal y como se pone de relieve, varios estudios han encontrado que las formas en que los profesionales de aprender y los métodos que utilizan durante el proceso de aprendizaje están relacionados con cambios en las prácticas profesionales (Goodwin y Kosnik, 2013). En esta línea Bakkenes et al. (2010) llevan a cabo un estudio longitudinal orientado a mejorar la comprensión de cómo aprenden los maestros, constando que las actividades de aprendizaje se asociaron significativamente con los resultados de aprendizaje, mientras que González-Arias et al. (2016) se plantean como objetivo construir y determinar las propiedades psicométricas de una escala de apreciación de la calidad de los programas de una asignatura, por parte de los estudiantes en un curso universitario.

Pero probablemente el campo de las nuevas tecnologías sea la temática más abordada. De los últimos trabajos realizados podemos destacar el desarrollado por Cobos-Sanchiz et al. (2016), con una experiencia de innovación docente universitaria con portafolios electrónicos, en formato blog, por parte del estudiantado y cuyos resultados evidencian, entre otras cuestiones, la utilidad del portafolio para el desarrollo de las competencias profesionales que el discente ha de adquirir. La tecnología robótica es abordada por Rodríguez et al. (2017) quienes llevan a cabo una intervención metodológica del tipo activa y cooperativa a través del uso de la tecnología robótica en la que intentan combinar el Aprender Haciendo con el Comprender como elementos necesarios para un aprendizaje significativo. Por su parte, Ausín et al. (2016) llevan a cabo una experiencia de innovación docente a través de Aprendizaje Basado en Proyectos (ABP) y la utilización de las Tecnologías de la Información y la Comunicación contextualizado en el grado de Pedagogía, concluyendo que la valoración más positiva está relacionada con el aprendizaje colaborativo y el trabajo en grupo. En estos aspectos profundiza Martínez (2017) quien diseña una guía didáctica basada en la integración de mundos virtuales y que se muestra eficaz para promover el aprendizaje colaborativo convirtiéndose en modelo para la integración de otras herramientas colaborativas en el entorno de la educación superior.

A pesar de todas estas iniciativas, se constata que la interdisciplinariedad es un campo poco abordado en las experiencias que pretenden ser innovadoras, pese a que tal y como afirman López et al. (2016) "el carácter integrador, transferible y multifuncional de las competencias requiere diseños y desarrollos curriculares más coordinados e interdisciplinares" (p.12), al mismo tiempo que se pone de relieve (Jacob, 2015) su importancia para ayudar a resolver algunas de las necesidades y desafíos de la educación superior actual.

Algunos trabajos que se centran en esta temática se dirigen a profundizar en cómo los estudiantes y responsables educativos entienden y apoyan la interdisciplinariedad en los departamentos (Shandas y Brown, 2016). Tomando como punto de partida las nuevas tecnologías, y más concretamente las wikis, Biasutti y EL-Deghaidy (2015) llevan a cabo un estudio centrado en el desarrollo de proyectos interdisciplinares para la escuela primaria con la ciencia como elemento nuclear que, al mismo tiempo, actuó como un centro de organización para la búsqueda de vínculos con otras disciplinas. Pharo et al. (2014) realizan un estudio de campo centrado en las comunidades de práctica con el objetivo de mejorar la enseñanza del cambio climático, implicando a profesorado de una amplia gama de disciplinas. Por su parte, Spitzer (2013) refleja el éxito de dos cursos interdisciplinares sobre ciencia, ingeniería y sociedad, al mismo tiempo que identifica una debilidad común: su posible desaparición si no se produce una integración de los temas interdisciplinarios en el curriculum de la formación.

Como se comprueba, son diversos los ámbitos trabajados desde planteamientos interdisciplinares. En el caso de la formación de maestros, coincidimos con Darling-Hammond y Richardson (2009) en que éstos deben aprender a enseñar de manera que desarrollen el pensamiento de orden superior y el rendimiento, lo que conlleva, necesariamente, vincular el curriculum de la formación. Desde este desafío se inicia el proyecto de innovación que presentamos en las páginas siguientes y cuya finalidad esencial radica en 
vincular la innovación con la interdisciplinariedad desde un tratamiento del curriculum de la formación desde un enfoque por competencias, puesto que tal y como afirman López et al. (2016), los estudios realizados hasta el momento sobre su evaluación son insuficientes, existiendo poco conocimiento sobre qué se está evaluando, cómo se está realizando esa evaluación o cuál es el impacto en el desarrollo competencial o en la mejora de la formación universitaria. En base a esto, la investigación en evaluación de competencias es un aspecto poco explorado en este proceso de cambio a pesar de ser un factor clave (López et al., 2016),

Tomando como referencia las aportaciones anteriormente referidas y con el objetivo de responder al cambio en las diferentes dimensiones, se inicia en el curso 2015-2016 en la Facultad de Formación del Profesorado de la Universidad de Santiago de Compostela (España), una experiencia de trabajo interdisciplinar entre tres de las cuatro asignaturas que conforman el tercer trimestre del segundo curso del Grado de Maestro en Educación Infantil: Proyectos e innovación Educativa, Diseño y Desarrollo Curricular; y Organización y Gestión del Aula. Nace así el Proyecto PODO (acrónimo de las tres materias: PrOyectos, Diseño, y Organización), con la finalidad de aunar esfuerzos cara el futuro en la formación inicial de los maestros, y cuyas características básicas describimos a continuación.

\section{MATERIALES Y MÉTODOS}

El diseño y desarrollo de este estudio se construye a partir de la formulación de los elementos relevantes en todo proceso investigador: objetivos a alcanzar, descripción de la metodología empleada y del instrumento, análisis del procedimiento seguido en la recogida de datos y por último, especificación de la muestra participante.

\section{Objetivos}

En el marco de este trabajo se contemplan los siguientes objetivos: a) aproximarse a la percepción global del desarrollo de las competencias en las materias que integran PODO; y b) realizar un análisis diferencial comparativo entre la situación antes de la experiencia y después de la experiencia.

\section{Metodología}

Con la opción metodológica se intentan superar algunas de las limitaciones señaladas en la literatura especializada, como por ejemplo evaluar únicamente el resultado final. Acorde a ello, se opta por un diseño pretest y postest que ofrezca oportunidad para medir el cambio en la autopercepción del alumnado con relación a su perfil competencial antes y después de la experiencia interdisciplinar. Se trata de un estudio cuantitativo basado en la técnica de encuesta, de corte no experimental y en el que no hubo manipulación de las variables independientes. El nivel de los análisis realizados tiene un alcance descriptivo, y considerando la distribución de la muestra se aplican pruebas no paramétricas en la línea de otros estudios realizados (Ausín et al.,2016; Contreras, 2016; González-Arias et al., 2016; Martínez, 2017; Shandas y Brown, 2016; Velthuis et al., 2014, entre otros).

\section{Instrumento}

Para la recogida de información se utilizó un cuestionario, al permitir gran versatilidad, eficiencia y posibilidad de generalización de los resultados de opinión de la población investigada sobre las variables objeto de estudio, con escala de medida tipo Likert y graduación de 5 puntos, instrumento frecuentemente utilizado en este tipo de estudios (Ausín et al., 2016; Contreras, 2016; González-Arias et al., 2016; Martínez, 2017). Una vez diseñado el instrumento se procedió a su validación de contenido en dos fases: en un primer momento, mediante un juicio de 5 expertos con experiencia contrastada en el ámbito de la formación del profesorado y el desarrollo competencial. Dichos expertos poseen un coeficiente de competencia que en todos los casos es superior a 0,8. En la siguiente fase se llevó a cabo una prueba piloto a 15 estudiantes de $2^{\circ}$ curso de la titulación del grado en maestro de educación primaria, a fin de determinar la comprensión y claridad de los enunciados, no teniendo la necesidad de realizar modificaciones al respecto. El análisis de los resultados determina que el instrumento se muestra como válido y fiable (Santos et al., 2016).

\section{Procedimiento}

La aplicación del cuestionario se llevó a cabo en dos momentos. La aplicación inicial o pre-test fue realizada el primer día de clase del primer cuatrimestre con todos los alumnos matriculados en las asignaturas vinculadas al proyecto y que asisten ese día a las sesiones presenciales. Posteriormente, se aplicó el instrumento en el segundo cuatrimestre, también en el primer día de clase, en el marco de otra materia de carácter obligatorio y cuyo docente se prestó a colaborar. Dado que había alumnos que no habían participado de la experiencia, se les solicitó que no cumplimentaran el cuestionario. Los análisis de la información se realizaron con el paquete estadístico SPSS 22.0. 


\section{Participantes}

La muestra, seleccionada con carácter intencional e indirecto, está compuesta por 55 y 54 estudiantes en la aplicación pre-test y post-test, respectivamente.

\section{RESULTADOS}

Los resultados obtenidos se presentan en dos subsecciones: en la primera se describe la percepción global del desarrollo de las competencias, y en la segunda se muestra el análisis diferencial comparativo entre el pre-test y post-test.

\section{Percepción global del desarrollo de las competencias}

El análisis del conjunto de respuestas múltiples nos permite conocer la valoración global inicial de partida de las competencias que el alumnado participante en proyecto indica poseer, lo cual nos permite comparar dicha valoración inicial con la que se percibe al finalizar el proyecto. Agrupamos las respuestas correspondientes a las valoraciones de "nada" y "poco" como un único conjunto, considerando dicha agrupación como puntuación baja, así como las respuestas en las valoraciones de "bastante" y "mucho", considerada puntuación alta. Las puntuaciones intermedias son tratadas igualmente. Comprobamos que las competencias vinculadas a las materias integradas en el proyecto se desarrollan considerablemente a juicio del alumnado, ya que globalmente el $60,6 \%$ de las valoraciones son altas al finalizar las materias, frente al $49,3 \%$ de las puntuaciones iniciales, tal y como podemos ver en la siguiente figura 1 .

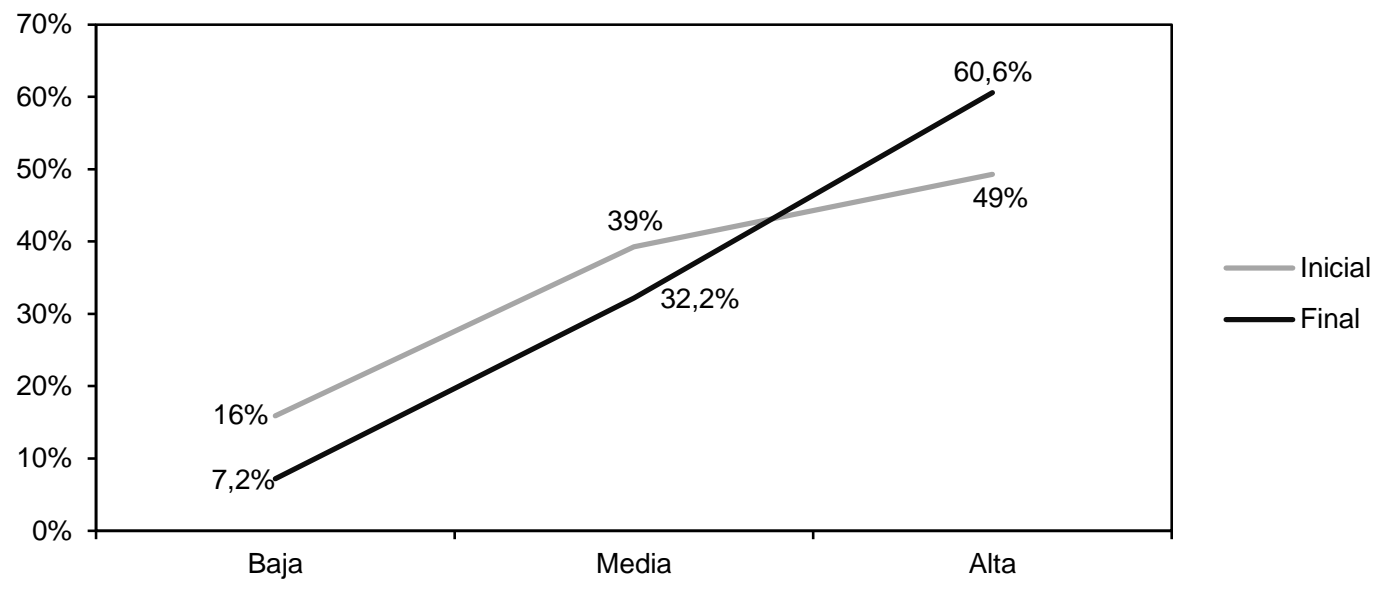

Fig. 1: Comparación de la distribución global de las competencias

Respecto a las medias obtenidas para cada variable en la aplicación inicial (Pre-test), podemos decir que prácticamente en todas ellas se incrementan en la aplicación final (Pos-test), salvo en aquellas referidas a las TICs y las que referencian aspectos relativos a los hábitos de salud, donde la media es menor al finalizar las asignaturas. En la tabla siguiente, se muestran los resultados.

Tabla 1: Estadísticos descriptivos para ambas aplicaciones

\begin{tabular}{|c|c|c|c|c|}
\hline \multirow[b]{2}{*}{ Variables (Competencias) } & \multicolumn{2}{|c|}{$\begin{array}{c}\text { Pre-test } \\
N=55\end{array}$} & \multicolumn{2}{|c|}{$\begin{array}{c}\text { Pos-test } \\
N=54\end{array}$} \\
\hline & Media & $\begin{array}{l}\text { Desviación } \\
\text { estándar }\end{array}$ & Media & $\begin{array}{l}\text { Desviación } \\
\text { estándar }\end{array}$ \\
\hline $\begin{array}{l}\text { CG_01 Conocer los objetivos, contenidos curriculares y } \\
\text { criterios de evaluación de la Educación Infantil. }\end{array}$ & 2,80 & 1,026 & 3,56 &, 572 \\
\hline $\begin{array}{l}\text { CG_02 Promover y facilitar los aprendizajes en la primera } \\
\text { infancia, desde una perspectiva globalizadora e integradora de } \\
\text { las diferentes dimensiones cognitiva, emocional, psicomotora y } \\
\text { volitiva. }\end{array}$ & 3,38 & ,828 & 3,41 & ,687 \\
\hline $\begin{array}{l}\text { CG_03 Diseñar y regular espacios de aprendizaje en contextos } \\
\text { de diversidad que atiendan a las singulares necesidades } \\
\text { educativas de los estudiantes, a la igualdad de género, a la } \\
\text { equidad y al respeto a los derechos humanos. }\end{array}$ & 3,18 & ,925 & 3,56 & ,718 \\
\hline
\end{tabular}


Tabla 1 (continuación)

\begin{tabular}{|c|c|c|c|c|}
\hline $\begin{array}{l}\text { CG_04 Fomentar la convivencia en el aula y fuera de ella y } \\
\text { abordar la resolución pacífica de conflictos. }\end{array}$ & 3,73 & ,732 & 3,91 & ,708 \\
\hline $\begin{array}{l}\text { CG_05 Saber observar sistemáticamente contextos de } \\
\text { aprendizaje y convivencia y saber reflexionar sobre ellos. }\end{array}$ & 3,24 & ,816 & 3,87 & ,825 \\
\hline $\begin{array}{l}\text { CG_06 Reflexionar en grupo sobre la aceptación de normas y el } \\
\text { respeto a los demás. }\end{array}$ & 3,82 & ,819 & 3,93 & ,773 \\
\hline $\begin{array}{l}\text { CG_07 Promover la autonomía y la singularidad de cada } \\
\text { estudiante como factores de educación de las emociones, los } \\
\text { sentimientos y los valores en la primera infancia. }\end{array}$ & 3,32 & 1,105 & 3,70 & ,838 \\
\hline $\begin{array}{l}\text { CG_08 Conocer las implicaciones educativas de las tecnologías } \\
\text { de la información y la comunicación y, en particular de la } \\
\text { televisión en la primera infancia. }\end{array}$ & 3,16 & ,764 & 2,69 & ,948 \\
\hline CG_09 Conocer fundamentos de dietética e higiene infantiles. & 3,56 & ,691 & 3,41 & ,836 \\
\hline $\begin{array}{l}\text { CG_10 Conocer fundamentos de atención temprana y las bases y } \\
\text { desarrollos que permiten comprender los procesos psicológicos, } \\
\text { de aprendizaje y de construcción de la personalidad en la primera } \\
\text { infancia. }\end{array}$ & 3,31 & ,836 & 3,20 & ,762 \\
\hline $\begin{array}{l}\text { CG_11 Conocer la organización de las escuelas de educación } \\
\text { infantil y la diversidad de acciones que comprende su } \\
\text { funcionamiento. }\end{array}$ & 2,80 & ,826 & 3,44 & ,904 \\
\hline $\begin{array}{l}\text { CG_12 Asumir que el ejercicio de la función docente ha de ir } \\
\text { perfeccionándose y adaptándose a los cambios científicos, } \\
\text { pedagógicos y sociales a lo largo de la vida. }\end{array}$ & 3,65 & ,844 & 3,87 & ,933 \\
\hline $\begin{array}{l}\mathrm{CG}_{1} 13 \text { Reflexionar sobre las prácticas de aula para innovar y } \\
\text { mejora la labor docente. }\end{array}$ & 3,31 & ,843 & 3,87 & 848 \\
\hline $\begin{array}{l}\text { CG_14 Adquirir hábitos y destrezas para el aprendizaje autónomo } \\
\text { y cooperativo y promoverlo en los estudiantes. }\end{array}$ & 3,44 & ,856 & 3,59 & ,880 \\
\hline $\begin{array}{l}\text { CE_1 Comprender que la dinámica diaria en educación infantil es } \\
\text { cambiante en función de cada estudiante, grupo y situación. }\end{array}$ & 3,98 & ,733 & 4,24 & ,799 \\
\hline CE_2 Ser flexible en el ejercicio de la función docente. & 3,83 & ,863 & 4,09 & ,708 \\
\hline $\begin{array}{l}\text { CE_3 Valorar la importancia de la estabilidad y la regularidad en } \\
\text { el entorno escolar, los horarios y los estados de ánimo del } \\
\text { profesorado como factores que contribuyen al progreso armónico } \\
\text { e integral de los estudiantes. }\end{array}$ & 3,53 & ,900 & 4,22 & 664 \\
\hline CE_4 Saber trabajar en equipo con otros profesionales. & 3,73 & ,827 & 3,85 & ,684 \\
\hline CE_5 Atender a las necesidades de los estudiantes. & 4,16 & ,714 & 4,41 & ,687 \\
\hline CT_1 Conocimiento instrumental de la lengua gallega. & 3,95 & ,951 & 3,78 & 1,040 \\
\hline $\begin{array}{l}\text { CT_2 Conocimiento instrumental de las tecnologías de la } \\
\text { información y de la comunicación. }\end{array}$ & 3,49 & ,767 & 3,33 & ,869 \\
\hline CT_3 Competencia informacional. & 3,27 & ,827 & 3,45 & ,774 \\
\hline
\end{tabular}

\section{Análisis diferencial comparativo: pre-test y post-test}

Nuestro objetivo se centra en conocer si existen diferencias significativas en la valoración del alumnado respecto al desarrollo de las competencias de las materias integradas en PODO. A fin de aplicar la prueba más adecuada para buscar dichas diferencias, inicialmente comprobamos si la muestra sigue una distribución normal. Con la prueba de Chi-cuadrado constatamos que la muestra en ambas aplicaciones, inicial y final, no sigue una distribución normal, ya que en ambos casos en todas las variables el valor $\mathrm{p}=0,000$. 
Tabla 2: Prueba de normalidad Chi-cuadrado

\begin{tabular}{|c|c|c|c|c|c|c|}
\hline \multirow{2}{*}{ Variables } & \multicolumn{3}{|c|}{ Pre-test } & \multicolumn{3}{|c|}{ Pos-test } \\
\hline & Chi-cuadrado & $g l$ & Sig. asintótica & Chi-cuadrado & $g l$ & Sig. asintótica \\
\hline CG_01 & 27,808 & 4 &, 000 & 72,189 & 4 &, 000 \\
\hline CG_02 & 38,769 & 4 & ,000 & 62,377 & 4 &, 000 \\
\hline CG_03 & 41,647 & 4 & ,000 & 61,623 & 4 &, 000 \\
\hline CG_04 & 45,500 & 4 &, 000 & 61,057 & 4 &, 000 \\
\hline CG_05 & 65,961 & 4 &, 000 & 40,679 & 4 &, 000 \\
\hline CG_06 & 34,731 & 4 &, 000 & 40,491 & 4 & ,000 \\
\hline CG_07 & 18,314 & 4 & ,001 & 34,642 & 4 & ,000 \\
\hline CG_08 & 39,731 & 4 & ,000 & 25,019 & 4 & ,000 \\
\hline CG_09 & 54,538 & 4 & ,000 & 51,846 & 4 & ,000 \\
\hline CG_10 & 38,962 & 4 & ,000 & 64,075 & 4 & ,000 \\
\hline CG_11 & 51,647 & 4 & ,000 & 32,000 & 4 & ,000 \\
\hline CG_12 & 32,038 & 4 & ,000 & 29,170 & 4 & ,000 \\
\hline CG_13 & 51,255 & 4 & ,000 & 30,868 & 4 & ,000 \\
\hline CG_14 & 29,731 & 4 & ,000 & 34,830 & 4 & ,000 \\
\hline CE_1 & 43,192 & 4 & ,000 & 50,491 & 4 & ,000 \\
\hline CE_2 & 57,725 & 4 & ,000 & 57,849 & 4 & ,000 \\
\hline CE_3 & 42,431 & 4 & ,000 & 54,453 & 4 & ,000 \\
\hline CE_4 & 35,692 & 4 & ,000 & 52,755 & 4 & ,000 \\
\hline CE_5 & 52,808 & 4 & ,000 & 58,415 & 4 & ,000 \\
\hline CT_1 & 29,731 & 4 & ,000 & 21,245 & 4 & ,000 \\
\hline CT_2 & 42,038 & 4 & ,000 & 45,774 & 4 & ,000 \\
\hline CT_3 & 34,346 & 4 & ,000 & 48,604 & 4 & ,000 \\
\hline
\end{tabular}

Así pues, al tratarse de muestras que no siguen una distribución de normalidad en ambos casos, recurrimos a pruebas no paramétricas para determinar si dichas diferencias son significativas. Aplicando $U$ de MannWhitney, los estadísticos de contraste indican que existen diferencias significativas en la valoración de algunas de las competencias genéricas y competencias específicas, tal y como podemos en la siguiente tabla. A través de la representación de los rangos vemos con claridad las diferencias entre los valores máximos y mínimos para cada variable en los diferentes momentos.

En las competencias en las cuales las diferencias son significativas ( $p=0,00$ considerando $p \leq 0,05$ ), la suma total de rangos (Figura 2) nos indican que las competencias genéricas que adquieren un mayor desarrollo al finalizar las asignaturas están todas ellas referidas a los contenidos contemplados en las mismas, adquiriendo una mayor relevancia los aspectos relacionadas con el conocimiento de objetivos, contenidos curriculares y criterios de evaluación (CG_01), donde el 55\% de las puntuaciones son asignadas a bastante/mucho al finalizar las materias, frente al $20 \%$ que inicialmente atribuye este grado de valoración.

Tabla 3: Estadísticos de la prueba U Mann-Whitney. a. Variable de agrupación: Pre-test y Pos-test

\begin{tabular}{|c|c|c|c|c|}
\hline Competencias & U de Mann-Whitney & W de Wilcoxon & Z & Sig. asintótica (bilateral) \\
\hline CG_01 & 714,00 & 2092,00 & $-4,49$ &, 00 \\
\hline CG_02 & 1276,00 & 2654,00 &,- 71 &, 47 \\
\hline CG_03 & 998,00 & 2376,00 & $-2,62$ &, 00 \\
\hline CG_04 & 1215,00 & 2593,00 & $-1,15$ &, 24 \\
\hline CG_05 & 753,00 & 2131,00 & $-4,29$ &, 00 \\
\hline CG_06 & 1285,00 & 2663,00 &,- 64 &, 52 \\
\hline CG_07 & 1070,50 & 2448,50 & $-2,06$ &, 03 \\
\hline CG_08 & 1027,00 & 2458,00 & $-2,38$ &, 01 \\
\hline
\end{tabular}


Tabla 3 (continuación)

\begin{tabular}{|c|c|c|c|c|}
\hline CG_09 & 1234,00 & 2665,00 & $-1,01$ &, 31 \\
\hline CG_10 & 1279,00 & 2710,00 &,- 70 &, 48 \\
\hline CG_11 & 892,50 & 2270,50 & $-3,36$ &, 00 \\
\hline CG_12 & 1179,50 & 2557,50 & $-1,34$ &, 17 \\
\hline CG_13 & 918,50 & 2296,50 & $-3,14$ &, 00 \\
\hline CG_14 & 1213,50 & 2591,50 & $-1,12$ &, 26 \\
\hline CE_1 & 1089,00 & 2467,00 & $-2,00$ &, 04 \\
\hline CE_2 & 1108,50 & 2486,50 & $-1,93$ &, 00 \\
\hline CE_3 & 757,50 & 2135,50 & $-4,23$ &, 53 \\
\hline CE_4 & 1289,00 & 2667,00 &,- 61 &, 09 \\
\hline CE_5 & 1138,50 & 2516,50 & $-1,68$ &, 40 \\
\hline CT_1 & 1253,50 & 2684,50 &,- 83 &, 42 \\
\hline CT_2 & 1264,00 & 2695,00 &,- 79 &, 16 \\
\hline CT_3 & 1178,00 & 2556,00 & $-1,38$ & \\
\hline
\end{tabular}

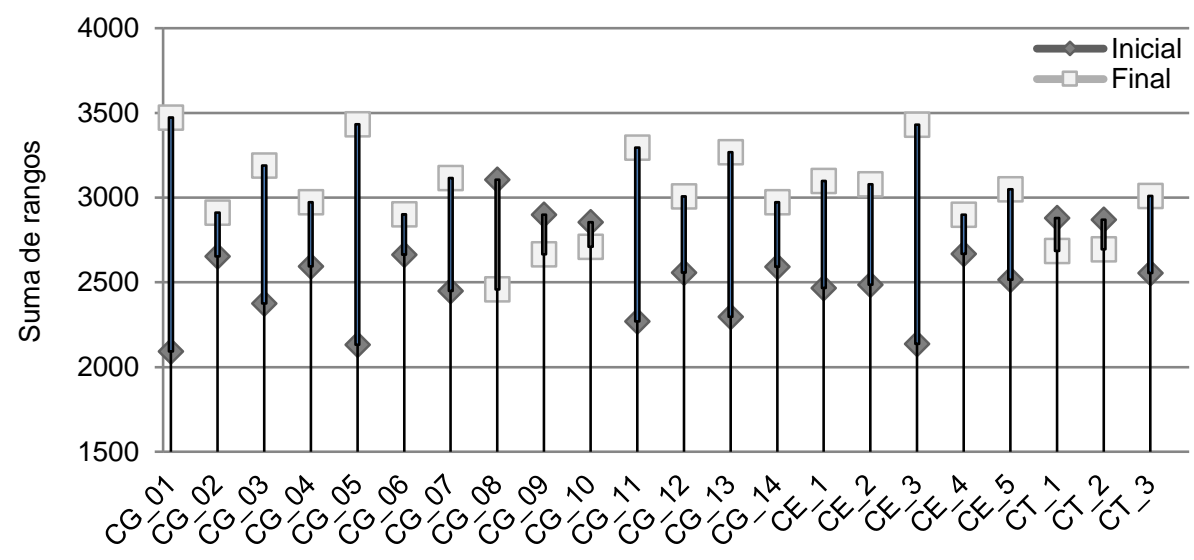

Fig. 2: Comparación del desarrollo de las competencias

Por otra parte, tal y como hemos comprobado, las diferencias también son significativas para los aspectos relacionados con el conocimiento de la organización de las escuelas de educación infantil y la diversidad de acciones que comprende su funcionamiento (CG_11), donde el $16,4 \%$ de las puntuaciones atribuidas inicialmente a una valoración alta, en el pos-test alcanzan el 42,6\% en el grado de valoración alta/muy alta.

Cabe señalar asimismo, los aspectos referidos a la observación sistemática en contextos de aprendizaje y convivencia (CG_05) donde el 31\% las puntuaciones atribuidas inicialmente a la mayor valoración en el desarrollo de dicha competencias llega hasta el 70,3\% al finalizar las materias integradas en PODO. Destacar también la competencia referida a promover la autonomía y la singularidad de cada estudiante como factores de educación de las emociones, los sentimientos y los valores en la primera infancia (CG_07). Aunque en este caso las diferencias son significativas, dichas diferencias son menores, ya que es el $4 \overline{1}, 5 \%$ de las puntuaciones alcanzadas en el pre-test atribuidas a la mayor valoración, frente al $61,1 \%$ alcanzado en el pos-test.

Mencionar también las competencias referida a reflexionar sobre las prácticas de aula para innovar y mejora la labor docente (CG_13), con diferencias significativas entre el 38,9\% de las valoraciones altas inicialmente y el $64,8 \%$ alcanzado al finalizar la experiencia. Los valores considerados en la graduación de la escala a la hora de comparar las diferencias son los correspondientes a las dos valoraciones superiores. Sin embargo las valoraciones intermedias también alcanzan en muchas ocasiones un incremento importante, aspecto este que podemos apreciar a través de la suma de rangos (Figura 2).

Referencia especial nos merecen las competencias referidas a las TICs, dado que aunque las diferencias son significativas, las valoraciones realizadas por el alumnado son posicionadas de forma negativa, es decir, inicialmente el alumnado consideraba en un porcentaje del 34,5\% tener un desarrollo alto/muy alto de dicha competencias, pero al finalizar las asignaturas integradas en el proyecto, dicha valoración disminuye, 
siendo tan sólo el 18,6\% alcanzado por dicho grado de valoración. La explicación a este hecho posiblemente esté justificada porque en los planes de estudio las materias con contenidos específicos de TICs, son cursadas en el último semestre, por lo que todavía no tienen conocimientos, sobre todo prácticos, de las aplicación de las TICs en el aula. Este hecho ocurre también con las variables referidas a aspectos de salud y dietética, si bien las diferencias son menores y en este caso no son significativas.

Respecto a las competencias específicas encontramos que tres de ellas presentan diferencias significativas (Tabla 3), presentándose la más acusada a la hora de valorar la importancia de la estabilidad y la regularidad en el entorno escolar, los horarios y los estados de ánimo del profesorado como factores que contribuyen al progreso armónico e integral de los estudiantes (CE_3), siendo el 49,1\% inicialmente atribuidas a alta/muy alta, frente al $87,1 \%$ alcanzado al final de la experiencia, no existiendo ninguna valoración asignada a baja/muy baja.

Igualmente las diferencias son significativas (Tabla 3) para las competencias referidas a comprender que la dinámica diaria en educación infantil es cambiante en función de cada estudiante, grupo y situación (CE_1), y la competencia referida a ser flexible en el ejercicio de la función docente (CE_2). Los porcentajes inicialmente alcanzados en el mayor grado de valoración se sitúan en torno al $75 \%$ inicial, frente al $87-$ $83,4 \%$ al finalizar la experiencia. Cabe destacar las altas puntuaciones asignadas a un grado de valoración medio, tanto en el pre-test como en el postest, situándose en ambos casos alrededor del $50 \%$, aspecto este que se puede constatar a través de la suma de rangos (Figura 2).

\section{DISCUSIÓN}

Con esta experiencia interdisciplinar se ha intentado superar la situación manifestada por Contreras (2016) cuando afirma que la innovación curricular no ha llegado a implementarse en las aulas provocando que la práctica siga atrapada en modelos tradicionales de enseñanza sustentados en clases magistrales, trabajando en pequeños seminarios o a través de bibliografía básica de la asignatura (Ausín et al., 2016). Desde nuestros planteamientos, el enfoque por competencias va más allá de posicionamientos retóricos 0 teóricos, ya que estamos firmemente convencidas de que puede constituirse en una ocasión privilegiada para promover una formación integral que ayude a vincular diferentes contenidos curriculares tanto desde una perspectiva inter como intradisciplinar. La opción por este modelo supone pues, una ocasión privilegiada para poner en marcha iniciativas que promuevan la actividad del alumnado en todos los niveles y situarlo, de esta forma, en el centro del proceso de enseñanza-aprendizaje.

El diseño y desarrollo de PODO ha estado orientado por unas premisas y principios que sirvieron de referencia a lo largo de todo el proceso. En primer lugar, y en coherencia con lo que se acaba de exponer, participamos del principio que defiende la necesidad de dar un mayor protagonismo al estudiante en sus procesos de formación, lo cual requiere ofrecer propuestas formativas significativas (López et al., 2016) que involucren trabajo colaborativo como potenciador de un aprendizaje significativo (Ausín et al., 2016). Por otra parte, se consideró especialmente buscar un equilibrio entre la actividad manual y la intelectual intentando superar lo denunciado por Rodríguez et al. (2017) cuando constata que en los últimos tiempos, el hacer ha sido sobrevalorado, dejando de lado el comprender.

Con la revisión de la literatura sobre el tema e investigaciones precedentes, hemos podido extraer unos puntos fuertes que se han trasladado a nuestro contexto, representando un valor añadido a este trabajo. Probablemente, el diseño pre-test y post-test, que ha posibilitado comparar el desarrollo competencial antes y después de la experiencia, sea la aportación más significativa, permitiendo hacer una evaluación fundamentada de la experiencia de innovación, pese a la dificultad que entraña esta tarea (Icarte y Labate, 2016; López et al., 2016). Sin embargo y pese a todo lo anterior, es posible identificar ciertos puntos débiles derivados de varios factores y que abren líneas de investigación. En primer lugar estaría el hecho de que estamos trabajando con percepciones acerca de las competencias y no con competencias directamente, lo que sin lugar a dudas es un indicador pero no una constatación del desarrollo competencial. En este sentido, sería conveniente llevar a la práctica nuevas experiencias curriculares que permitan al alumnado la capacidad de aplicar un saber, una habilidad o unas pautas de comportamiento, y que constituyen la esencia de las competencias.

Por otra parte, la evaluación inicial del nivel de dominio competencial del alumnado participante en PODO sitúa su nivel de autorpercepción en un nivel medio o alto. ¿De dónde proviene esta percepción del grado de desarrollo de sus competencias docentes? Es posible que de haber cursado ya un año de formación inicial, o del aprendizaje vicario llevado a cabo en su escolarización previa, de una inquietud personal que promueva una autoformación, o incluso de su propia conceptualización acerca de las competencias, como ya se puso de manifiesto en trabajos previos (Villaroel y Bruna, 2017). Al hilo de esto surge una pregunta no carente de importancia: ¿habría sido el mismo nivel de percepción de desarrollo de las competencias 
consideradas fuera del marco del Proyecto? Es decir, ¿ha contribuido realmente PODO como experiencia interdisciplinar a un incremento competencial que no hubiera tenido lugar sin ella?

Sin duda, existen muchas explicaciones plausibles pero que se sitúan en el terreno de las hipótesis y a las que no podemos dar respuesta en este momento con los datos de los que disponemos, lo que hace necesario la realización de estudios complementarios. Una posibilidad estaría en el análisis de contenido de e-portafolios, en la línea de investigaciones anteriores (Cobos-Sanchiz et al., 2016) cuyo análisis ayudaría a obtener una visión más holística y explicativa. Junto a esto, consideramos necesario profundizar en el objeto de estudio a través de una metodología mixta de investigación en la que la perspectiva cuantitativa y cualitativa se complementen, en la línea de estudios precedentes y que permita no sólo cuantificar sino, y sobre todo, comprender las motivaciones que están detrás de los datos de cara a una mejora de la formación inicial del profesorado.

Evidentemente, son muchas las posibilidades que se abren. Lo que parece demostrado, desde la revisión de la literatura así como de la evaluación de nuestra experiencia, es que este tipo de iniciativas pueden ayudar a conseguir en las aulas de formación universitaria un cambio que supere la dimensión formal y ayuden a superar la lógica academicista y disciplinar que, de forma generalizada, ha primado hasta no hace demasiado tiempo en estas enseñanzas. Sin embargo, debemos tener en cuenta que la profundidad y el tiempo de experiencia en la enseñanza basada en competencias es un elemento clave para una transformación de las prácticas institucionalizadas (Bustamante et al., 2017). Por lo tanto, habrá que dar tiempo al tiempo y continuar en esta línea de trabajo para, desde una dimensión longitudinal, tener una visión más ajustada a la realidad de nuestro objeto de estudio.

\section{CONCLUSIONES}

Los objetivos pretendidos con este trabajo se centraban en los siguientes: a) aproximarse a la percepción global del desarrollo de las competencias en las materias que integran PODO; y b) realizar un análisis diferencial comparativo entre la situación antes de la experiencia y después de la experiencia.

1) Los resultados obtenidos con el análisis de los datos muestran que la percepción del alumnado al finalizar las materias del tercer cuatrimestre se sitúan en el $60,6 \%$ los que las consideran altas, frente al $49,3 \%$ que se manifestaba en este sentido al inicio del cuatrimestre.

2) La experiencia llevada a cabo evidencia aspectos sobre el desarrollo de competencias propias de la titulación y de las materias, que posiblemente de forma individual en cada materia no sería posible.

3) El desarrollo de las competencias, es más notorio en aquéllas directamente relacionadas con los aspectos básicos referidos fundamentalmente al conocimiento de la profesión docente, y más concretamente del aula de Educación Infantil y el diseño y desarrollo del currículum.

4) Los aspectos referidos a la planificación docente son los que a través de la experiencia se han desarrollado en mayor medida, así como los aspectos propios del contexto dentro aula referidos fundamentalmente a la organización, espacios, observación, etc...

5) Pese a que hemos podido comprobar que aquellas competencias relacionadas con la salud infantil, así como la estimulación temprana, no sólo no se han desarrollado, sino que la experiencia no ha colaborado en ello, disminuyendo la percepción del alumnado en el desarrollo de la misma a lo largo del proyecto, creemos que los resultados confirman que el trabajo interdisciplinar ha sido una buena opción de sumar esfuerzos de cara al desarrollo competencial de los maestros de Educación Infantil.

\section{REFERENCIAS}

Alvarado-Lagunas, E., Luyando-Cuevas, J.R. y Picazzo-Palencia, E., Percepción de los estudiantes sobre la calidad de las universidades privadas en Monterrey, https://goo.gl/3j4uXE, ISSN: 2007-2872, Revista Iberoamericana de Educación Superior (RIES), 6(17), 58-76 (2015)

Ausín, V., Abella, V., Delgado, V., y Hortigüela, D., Aprendizaje Basado en Proyectos a través de las TIC: Una Experiencia de Innovación Docente desde las Aulas Universitarias, doi: https://dx.doi.org/10.4067/S0718-50062016000300005, Formación Universitaria, 9(3), 31-38 (2016)

Avalos, B., Teacher professional development in Teaching and Teacher Education over ten years, doi: http://dx.doi.org/10.1016/j.tate.2010.08.007, Teaching and Teacher Education, 27(1), 10-20 (2011) 
Bakkenes, I., Vermunt, J. D., y Wubbels, T., Teacher learning in the context of educational innovation: Learning activities and learning outcomes of experienced teachers, doi: 10.1016/j.learninstruc.2009.09.001, Learning and Instruction, 20, 533-548 (2010)

Barber, M. y Mourshed, M., How the world's best-performing school systems come out on top (en línea: https://goo.gl/nfnDQw), acceso: 17 de enero de 2011, McKinsey\&Company, Bélgica (2007)

Biasutti, M. y EL-Deghaidy, H., Interdisciplinary project-based learning: an online wiki experience in teacher education, doi: http://dx.doi.org/10.1080/1475939X.2014.899510, Technology, Pedagogy and Education, 24(3), 339-355 (2015)

Bustamante, M. A., Lapo, M. C., Oyarzún, C. H. y Campos, R. M., Análisis de la Percepción del Docente en Tres Universidades Chilenas tras la Implementación del Currículum Basado en Competencias, doi: http://dx.doi.org/10.4067/S0718-50062017000400009, Formación Universitaria, 10(4), 97-110 (2017)

Cobos-Sanchiz, D., López-Meneses, E., y Llorent-Vaquero, M., Propuesta de Innovación Didáctica con Portafolios Electrónicos en Formato Blog: Un Caso de Estudio en una Universidad, doi: https://dx.doi.org/10.4067/S0718-50062016000600004, Formación Universitaria, 9(6), 27-42 (2016)

Contreras, S.A., Pensamiento Pedagógico en la Enseñanza de las Ciencias: Análisis de las Creencias Curriculares y sus Implicancias para la Formación de Profesores de Enseñanza Media, doi: https://dx.doi.org/10.4067/S0718-50062016000100003, Formación Universitaria, 9(1), 15-24 (2016)

Darling-Hammond, L., y Richardson, N., Research review/teacher learning: What matters? https://goo.gl/VkLXyw, ISSN: 0013-1784, Educational Leadership, 66, 46-53 (2009)

Davis, S.N. y Jacobsen, S.K., Curricular Integration as Innovation: Faculty Insights on Barriers to Institutionalizing Change, doi: 10.1007 / s10755-013-9254-3, Innovative Higher Education, 39(1), 17-31 (2014)

De la Cerda Toledo, M., Mentoring and Service Learning in the University: An Experience Organised by the Faculty of Pedagogy of the University of Barcelona, doi: 10.12691/education-2-8A-1, American Journal of Educational Research, 2(8A), 1-7 (2014)

Desimone, L.M, A primer on effective professional development, doi: doi/abs/10.1177/003172171109200616, Phi Delta Kappan, 92(6), 68-71 (2011)

European Comission, Teachers' Professional Development - Europe in international comparison - An analysis of teachers' professional development based on the OECD's Teaching and Learning International Survey (TALIS), Luxemburgo, Oficina de Publicaciones Oficiales de la Unión Europea, Bélgica (2010)

EURYDICE y CEDEFOP, Structures des systèmes d'enseignement et de formation initiale dans l'Union Européenne, Luxemburgo, Oficina de Publicaciones Oficiales de la Unión Europea, Bélgica (1995)

Fernández-Fernández, S., Arias-Blanco, J.M., Fernández-Alonso, R. Burguera-Condon, J. y FernándezRaigoso, M., Reflective and Inquary Thinking in Education. Aspects to consider in teacher education, doi: http://dx.doi.org/10.7203/relieve.22.2.8425, RELIEVE, 22(2), art. 3 (2016)

Girvan, C., Conneely, C. y Tangney, B., Extending experiential learning in teacher professional development, doi: http://dx.doi.org/10.1016/j.tate.2016.04.009, Teaching and Teacher Education, 58, 129-139 (2016)

González-Arias, M.I., Carabantes Olivares, E. y Muñoz-Carreño, N.E., Construcción y Validación de la Escala de Apreciación de la Calidad del Programa de Asignatura: Propuesta para el Estudio de la Calidad de la Docencia, doi: https://dx.doi.org/10.4067/S0718-50062016000100009, Formación Universitaria, 9(1), 77-90 (2016)

Goodwin, A.L. y Kosnik, C., Quality teacher educators = quality teachers? Conceptualizing essential domains of knowledge for those who teach teachers, doi: http://dx.doi.org/10.1080/13664530.2013.813766, Teacher Development, 17(3), 334-346 (2013)

Icarte, G.A., y Labate, H.A., Metodología para la Revisión y Actualización de un Diseño Curricular de una Carrera Universitaria Incorporando Conceptos de Aprendizaje Basado en Competencias, doi: https://dx.doi.org/10.4067/S0718-50062016000200002, Formación Universitaria, 9(2), 03-16 (2016)

Jacob, W.J., Interdisciplinary Trends in Higher Education, doi: http://dx.doi.org/10.1057/palcomms.2015.1, Palgrave Communications, 1, 15001-15005 (2015)

López, C., Benedito, V., y León, M.J., El Enfoque de Competencias en la Formación Universitaria y su Impacto en la Evaluación: La Perspectiva de un Grupo de Profesionales Expertos en Pedagogía, doi: https://dx.doi.org/10.4067/S0718-50062016000400003, Formación Universitaria, 9(4), 11-22 (2016) 
Martínez, G.A., Diseño de una Guía Didáctica basada en la Integración de Mundos Virtuales al Entorno Educativo de la Universidad de Cundinamarca, doi: https://dx.doi.org/10.4067/S0718-50062017000100002, Formación Universitaria, 10(1), 3-14 (2017)

Pharo, E., Davison, A., McGregor, H., Warr, K. y Brown, P., Using communities of practice to enhance interdisciplinary teaching: lessons from four Australian institutions, http://dx.doi.org/10.1080/07294360.2013.832168, Higher Education Research \& Development, 22(2), 341-354 (2014)

Renta-Davids, A.I., Jiménez-González, J.M., Fandos-Garrido, M. y González-Soto, A.P., Organisational and training factors affecting academic teacher training outcomes, doi: http://dx.doi.org/10.1080/13562517.2015.1136276, Teaching in Higher Education, 21(2), 219-231 (2016)

Rodríguez, A.B, Ramírez, L.J. y Fernández, W., Metodologías Activas para Alcanzar el Comprender, doi: https://dx.doi.org/10.4067/S0718-50062017000100009, Formación Universitaria, 10(1), 79-88, (2017)

Santos, M.C., Sarceda-Gorgoso, M.C. y Rial, P., Validación de un instrumento para evaluar competencias genéricas en la formación del maestro de Educación Infantil, https://goo.gl/EdXYnM, ISSN: 1390-969X, Ecos de la Academia, Revista de la Facultad de Educación, Ciencia y Tecnología, FECYT, Ibarra, Ecuador, Universidad Técnica del Norte, 3, 142-155 (2016)

Sarceda-Gorgoso, M.C. y Rodicio-García, M.L., Escenarios formativos y competencias profesionales en la formación inicial del profesorado, Revista Complutense de Educación, 24(1), 35-52 (en prensa)

Shandas, V. y Brown, S.E., Toward an Interdisciplinary Agenda in Higher Education: Empirical evidence from the field of urban and regional planning, doi:10.1007/s10755-016-9362-y, Innovative Higher Education, 41(411) (2016)

Spitzer, H., Introduction of Interdisciplinary Teaching: Two Case Studies, doi: 10.1007/s11948-013-9475$y$, Science and Engineering Ethic, 19(4), 1451-1454 (2013)

Velthuis, C., Fisser, P. y Pieters, J., Teacher Training and Pre-service Primary Teachers' Self-Efficacy for Science Teaching, doi: 10.1007/s10972-013-9363-y, Journal of Science Teacher Education, 25(4), 445464 (2014)

Villarroel, V.A. y Bruna, D.V., Pedagogical Competencies of University Teachers: A Case Study, Which Incorporates the Perspective of Chilean Teachers and Students, doi: http://dx.doi.org/10.4067/S071850062017000400008, Formación Universitaria, 10(4), 75-96 (2016)

Zeichner, K., Rethinking the connections between campus courses and field experiences in college- and university-based teacher education, doi: https://doi.org/10.1177/0022487109347671, Journal of Teacher Education, 61, 89-99 (2010) 